\title{
Revisión de las intervenciones que mejoran la utilidad percibida del aprendizaje de los estudiantes
}

\author{
Javier Sánchez-Rosas ${ }^{1}{ }^{*}$ \\ https://orcid.org/0000-0002-7669-8981. \\ ${ }^{1}$ Universidad Nacional de Córdoba, Córdoba, Argentina. \\ Pablo Sebastián Correa ${ }^{1},{ }^{* *}$ \\ https://orcid.org/0000-0001-7597-7180 \\ "Instituto de Investigaciones Psicológicas (IIPsi-CONICET-UNC), Córdoba, Argentina. \\ Iván Daniel Díaz ${ }^{1}, * * *$ \\ https://orcid.org/0000-0001-9254-7182 \\ Universidad Nacional de Córdoba, Córdoba, Argentina
}

Recibido: 28/12/2018 Revisado: 15/02/2019 Publicado: 15/12/2019

\begin{abstract}
Resumen
Actualmente, los docentes se enfrentan al desafío de lograr que los estudiantes perciban como útiles los contenidos que enseñan en clase. Desde la teoría expectativa-valor de la motivación, la utilidad percibida es considerada flexible a las intervenciones externas. Aunque un cuerpo reciente y creciente de investigaciones han evaluado la efectividad de tales intervenciones, no se conocen publicaciones que hayan revisado la cantidad, características, conceptos, correlatos y funcionamiento de las intervenciones investigadas. Para tal fin, en este trabajo se buscó describir las intervenciones que promueven la utilidad percibida por los estudiantes. Se llevó a cabo una revisión bibliográfica no sistemática, construyéndose criterios de análisis ad hoc. Se identificaron doce intervenciones, todas basadas en el modelo teórico de expectativa-valor. El tipo, cantidad y duración de las actividades es diverso y las mismas consisten en pedir a los estudiantes que hagan conexiones de los temas aprendidos en clase con su vida cotidiana. Asimismo, la frecuencia de dichas conexiones es el mecanismo a través del cual las intervenciones poseen efectos sobre el aprendizaje. Esta revisión aporta a la práctica e investigación en educación una diversidad de recursos para producir cambios en la utilidad percibida y mejorar el interés y el rendimiento de los estudiantes. Palabras clave: rendimiento académico; aprendizaje; motivación; interés; psicología de la educación
\end{abstract}

\section{Review of the Interventions that Improve the Perceived utility of Learning by Students}

\footnotetext{
Abstract

Currently, educators face the challenge of making students consider useful what they teach in class. According to the expectation-value of motivation theory, the perceived usefulness is considered flexible in the face of external interventions. Even though a recent growing body of research has assessed the effectiveness of such interventions, there are no known publications that have reviewed the quantity, characteristics, concepts, correlates, and functioning of the examined interventions. To this end, the intention of this study was to describe the interventions that promote the usefulness perceived by the students. A non-systematic bibliographical review was carried out, producing ad hoc analysis criteria. Twelve
} 
interventions were identified, all based on the theoretical expectation-value model. The type, quantity and duration of the activities are diverse, consisting of asking students to make connections between the topics learned in class and their daily lives. Furthermore, the frequency of those connections is the mechanism through which interventions have an effect on learning. This review provides a variety of resources to the practice and research on education in order to produce changes in the perceived usefulness and improve the student's focus and performance.

Keywords: Academic achievement, learning, motivation, focus, educational psychology

\title{
Revisão das intervenções que melhoram a percepção da utilidade da aprendizagem dos estudantes
}

\begin{abstract}
Resumo
Atualmente, os docentes enfrentam o desafio de fazer com que os estudantes percebam como úteis os conteúdos ensinados na sala de aula. Desde a teoria expectativa-valor da motivação, a utilidade percebida é considerada como flexível diante das intervenções externas. Embora um número crescente e recente de pesquisas tenha avaliado a efetividade de tais intervenções, não há ciência de publicações que tenham revisado a quantidade, características, conceitos, correlatos e funcionamento das intervenções pesquisadas. Este trabalho procurou descrever as intervenções que promovem a utilidade percebida pelos estudantes. Realizou-se uma revisão bibliográfica não sistemática, a partir da construção de critérios de análise ad hoc. Identificaram-se doze intervenções, todas elas baseadas no modelo teórico de expectativa-valor. O tipo, quantidade e duração das atividades diferem. Tais atividades consistem em pedir aos estudantes que façam conexões dos temas aprendidos na sala de aula com a sua vida cotidiana. Da mesma forma, a frequência de tais conexões é o mecanismo pelo qual as intervenções possuem efeitos sobre a aprendizagem. Esta revisão contribui para a prática e a pesquisa em educação com uma diversidade de recursos para produzir mudanças na percepção da utilidade dos conteúdos aprendidos e melhorar o interesse e rendimento dos estudantes.
\end{abstract}

Palavras-chave: rendimento acadêmico; aprendizagem; motivação; interesse; psicologia da educação

Como citar:

Sanchez-Rosas, J., Correa, P.S., \& Díaz, I.D. (2019). Revisión de las intervenciones que mejoran la utilidad percibida del aprendizaje de los estudiantes. Revista Digital de Investigación y Docencia Universitaria, 13(2), 45-56. https://doi. org/10.19083ridu.2019.1077

$\mathrm{M}$ ejorar la motivación y el aprendizaje en los ámbitos académicos es una meta compartida por todos los docentes, las instituciones y las políticas educativas. Alcanzar este objetivo es relevante si se tiene en cuenta los resultados obtenidos de las pruebas APRENDER (Secretaria de Evaluación Educativa, 2017) en Argentina, en las cuales el 18\% de los estudiantes de secundaria se encuentra en un nivel por debajo del básico en Lengua, mientras que en Matemáticas esta cifra asciende hasta el 41\%. A su vez, el
Programa para la Evaluación Internacional de Alumnos (Organización para la Cooperación y el Desarrollo Económico [OCDE], 2015) indicó en su último informe que, en países como Brasil (44.1\%), México (33.8\%), Estados Unidos (13.6\%) y España (10.3\%), los estudiantes poseen un bajo rendimiento en Matemáticas, Lengua y Ciencias. En los estudiantes de nivel medio y aquellos que se inician en los estudios superiores, uno de los factores que interfieren en la motivación, el aprendizaje, el rendimiento, la deserción y la repitencia escolar, es la 
escasa utilidad percibida por los estudiantes de los contenidos y actividades de aprendizaje (Anderman \& Maehr, 1994; Hulleman \& Harackiewicz, 2009; Lepper, Corpus, \& Iyengar, 2005).

La utilidad percibida ha sido estudiada en el marco de una de las teorías más prominentes en el estudio de la motivación y el aprendizaje: por ejemplo, en la teoría de expectativa-valor propuesta por Eccles et al. (1983), se propone que la motivación en contextos educacionales está determinada por las expectativas de éxito en una actividad y las creencias sobre la importancia percibida de la misma. Entre las creencias de valor del modelo, el concepto valor de la tarea adquiere un rol importante ya que es considerado un predictor importante del rendimiento y las elecciones de los estudiantes (Eccles et al., 1983; Simpkins, Davis-Kean, \& Eccles, 2006; Wigfield \& Cambria, 2010; Wigfield \& Eccles, 2000; Xiang, Bruene, \& Chen, 2005).

El valor de la tarea es entendido como el incentivo para comprometerse en actividades académicas (Eccles et al., 1983; Wigfield \& Eccles, 2000) y está integrado por cuatro componentes: importancia (relevante para la propia identidad), interés (disfrute inherente de la tarea), costo (barreras psicológicas para, y consecuencias negativas de comprometerse en una tarea) y utilidad (útil para la consecución de metas a corto y largo plazo). La importancia y el interés poseen un carácter intrínseco y, en consecuencia, esta condición los haría más difícil de manipular extrínsecamente. Según Harackiewicz, Canning, Tibbetts, Priniski y Hyde (2016), esta sería la razón por la que los investigadores han abordado más frecuentemente el componente utilidad, ya que presenta mejores posibilidades de ser modificado a través de intervenciones.

Las intervenciones en el valor de utilidad (UVI, utility-value intervention, por su sigla y expresión en inglés) son procedimientos prácticos que, bajo diversas modalidades o aplicados a diferentes personas, se han implementado para mejorar la percepción de los estudiantes acerca de lo aprendido en clase. En general, estas intervenciones consisten en buscar que los estudiantes relacionen un tema o material de aprendizaje con sus propias vidas (Hulleman \& Harackiewicz, 2009). Algunos estudios han mostrado que estas inter- venciones producen un incremento en el interés y el rendimiento en diferentes temas o materias (Canning et al., 2018; Cook, Purdie-Vaughns, Garcia, \& Cohen, 2012; Durik \& Harackiewicz, 2007; Durik, Vida, \& Eccles, 2006; Harackiewicz, Durik, Barron, Linnenbrink-Garcia, \& Tauer, 2008; Hulleman, Godes, Hendricks, \& Harackiewicz, 2010; Hulleman \& Harackiewicz, 2009; Lazowski \& Hulleman, 2016; Rosenzweig et al., 2018; Shechter, Durik, Miyamoto, \& Harackiewicz, 2011; Shin et al., 2019; Walton, 2014).

Si bien existe consenso sobre la influencia de diferentes variables motivacionales (Karadag, 2017) y métodos instruccionales (Hattie, 2008, 2012) sobre el rendimiento académico de los estudiantes, no se encontraron reportes de investigación que describan aquellas intervenciones que han buscado mejorar la utilidad percibida por los estudiantes y/o que, en última instancia, incidan sobre el aprendizaje y el rendimiento. Tal revisión les permitiría a docentes universitarios, contar con un panorama de posibles acciones a implementar para mejorar la utilidad percibida por los estudiantes de los contenidos y actividades de aprendizaje. En función de esto, en el presente trabajo se propuso describir las intervenciones que promueven la utilidad percibida por los estudiantes (Hulleman \& Harackiewicz, 2009). Para ello, se identificaron las intervenciones existentes y se caracterizaron los procedimientos y actividades de las mismas. Además, se describieron los efectos de las intervenciones sobre la utilidad percibida en sí y sobre otras variables.

Antes de proceder al desarrollo de la revisión efectuada, se describen algunos aspectos metodológicos que guiaron y estructuraron a la misma. En el presente trabajo se efectuó una revisión bibliográfica no sistemática de investigaciones empíricas que evaluaron intervenciones cuyo fin era mejorar la utilidad percibida por los estudiantes. Se analizó cada investigación de manera descriptiva, sin apelar a procedimientos estadísticos para su análisis.

La estrategia de búsqueda de la información bibliográfica consistió en la utilización de palabras clave en inglés (utility value, intervention, utility intervention, expectancy-value) y en español (valor de utilidad, intervención, intervención de utilidad, expectativa-valor). La elección de las mismas se basó en el interés por explorar aque- 
llas intervenciones que se sustenten en el modelo teórico de expectativa-valor. Asimismo, se empleó la sigla STEM (Science, Technology, Engineering and Mathematics) dado que, se pudo anticipar la existencia de diversas intervenciones que han mejorado la percepción de utilidad en materias o carreras de ciencias naturales, tecnología, ingeniería y matemáticas.

La búsqueda se realizó en las bases de datos: Dialnet, Science Direct y la Biblioteca Virtual del Ministerio de Ciencia y Tecnología de Argentina, la cual incluye a bases como de datos como Ebscohost, Scopus o Scientific Electroni Library Online (Scielo). Se aplicaron filtros que, específicamente, proveyeron artículos de investigación en inglés y español relacionados al área de psicología educacional, que poseían textos completos. Además, se implementó una búsqueda manual en el listado de referencias de cada artículo encontrado para identificar trabajos que no hubieran sido detectados por la búsqueda en bases de datos. La búsqueda se hizo en el mes de diciembre del año 2017. La estrategia empleada permitió encontrar un total de 64 documentos.

Se eligieron solo aquellos artículos empíricos que tenían por objetivo, o como uno de sus objetivos, diseñar y evaluar intervenciones para modificar la utilidad percibida y que las mismas se encuentren basadas en el modelo teórico de expectativa-valor. Se procuró excluir publicaciones bajo la modalidad de ponencias en congreso, capítulos de libro y sin revisión por pares con el fin de considerar sólo artículos publicados en revistas periódicas que permitan acceder a información metodológica sobre las intervenciones de forma más detallada. A su vez, las ponencias en congresos fueron descartadas porque su contenido suele ser previo al que compone una publicación posterior, lo cual implicaría contar con documentos duplicados en el análisis. La aplicación de estos criterios resultó en un total de doce artículos empíricos que conforman el conjunto del material a describir en tanto se los considera pertinentes para los objetivos del presente trabajo.

Con el fin de responder a los objetivos planteados anteriormente, de la información relevada se identificaron autores y año de cada publicación. Luego se describieron los procedimientos y consignas utilizadas en cada intervención. Posterior- mente, se caracterizaron las actividades que componían a cada procedimiento implementado, para ello se crearon cinco categorías para organizar la información: tipo, cantidad, características, duración y frecuencia de cada actividad. Por último, se extrajeron de las fuentes de información las variables antecedentes o simultáneas a la manipulación de la utilidad y los efectos (dirección y magnitud) de la intervención sobre variables consecuentes.

Cabe señalar que, la información recabada se ordenó considerando el nivel educativo de las muestras de cada estudio. Concretamente, en primer lugar, se exponen los datos y el análisis efectuado sobre aquellos estudios que han empleado muestras de estudiantes de nivel secundario. En segundo lugar, se desarrolla la información obtenida de los estudios que han recurrido a muestras universitarias. La organización de los datos y el análisis efectuado se realizó de forma simultánea y en conjunto por los autores de este artículo.

\section{Caracterización de los procedimientos de las intervenciones en utilidad percibida}

Con frecuencia, los investigadores que han diseñado estas intervenciones informan, con cierto grado de precisión, en la sección metodológica, los procedimientos implementados y que el lector interesado podrá consultar en cada publicación. De manera breve, se describen a continuación los diferentes procedimientos de las intervenciones tendientes a modificar la utilidad percibida por los estudiantes. La descripción incluye aquellas intervenciones diseñadas para mejorar la utilidad percibida pero que no informan sus efectos sobre la utilidad misma y las que sí han informado sus efectos sobre la utilidad percibida y otras variables consecuentes. En la tabla 1 se encuentra un resumen de la información recolectada.

En muestras con estudiantes de nivel secundario, algunas intervenciones consisten en proponerle a un grupo de estudiantes que escriban un ensayo sobre cómo vincular o aplicar lo aprendido en clase a sus propias vidas o a la vida de una persona conocida (Durik et al., 2014; Hulleman \& Harackiewicz, 2009). En otros estudios se ha recurrido a la inclusión de los padres de los participantes, concretamente a través de folletos, sitios web informativos y entrevistas telefónicas, se le brinda consejos y herramientas para que comenten y les 
muestren a sus hijos la utilidad de aprender sobre temas como las matemáticas o las ciencias naturales, tanto para sus vidas cotidianas como para su futuro profesional (Harackiewicz et al., 2012; Hyde et al., 2016; Rozek et al., 2015, 2017).

Entre las investigaciones que han empleado estudiantes universitarios como participantes de sus muestras, una de las formas en la que diversos estudios han elaborado intervenciones en pos de mejorar la utilidad percibida también reside en proponerle a un grupo de estudiantes que escriban un ensayo, resumen o carta sobre cómo relacionar o aplicar temas aprendidos en clases a sus propias vidas o a la vida de una persona cercana a ellos (Harackiewicz et al., 2016; Hulleman et al., 2017). Otra alternativa posible es pedirles a los estudiantes que realicen búsquedas de información en sitios web sobre los temas que aprenden y redacten también en formato de carta o ensayo por qué es útil o importante lo aprendido en clase (Hulleman et al., 2010).

Además, algunos estudios han empleado ejercicios con el fin de que los temas aprendidos en clase puedan ser percibidos como útiles para desarrollar una carrera a futuro. Estas intervenciones han recurrido al uso de videos en computadora o ejemplos sobre la utilidad a corto y largo plazo de las temáticas desarrolladas en el aula (Shechter et al., 2011), o han empleado historias ficticias en las cuales un estudiante conecta lo aprendido en clase con sus aspiraciones futuras y posteriormente se les pide a los estudiantes que intenten visualizar el material de estudio como lo hace el personaje de la historia (Lee Johnson \& Sinatra, 2012). También, se han implementado intervenciones en las cuales el docente intenta demostrar el valor que posee una temática particular para resolver problemáticas socialmente relevantes (Brown et al., 2015).

\section{Talbla 1}

Descripción de las intervenciones sobre utilidad percibida

\begin{tabular}{|c|c|c|c|c|}
\hline Autores & $\begin{array}{c}\text { Muestra } \\
\text { (Nivel educativo) }\end{array}$ & Actividad & Extensión & Duración \\
\hline $\begin{array}{l}\text { Hulleman \& } \\
\text { Harackiewicz (2009); } \\
\text { Hulleman et al. (2017) }\end{array}$ & Estudiantes universitarios & $\begin{array}{l}\text { Ensayos (8), mapas } \\
\text { conceptuales, folletos }\end{array}$ & $\begin{array}{l}\text { Al menos cinco ora- } \\
\text { ciones }\end{array}$ & $2^{\circ}$ semestre \\
\hline Hulleman et al., (2010) & Estudiantes universitarios & Resumen (1), cartas (1) & $\begin{array}{l}\text { Al menos tres pár- } \\
\text { rafos }\end{array}$ & 15 semanas \\
\hline Shechter et al., (2011) & Estudiantes universitarios & Audiovisuales & $\mathrm{s} / \mathrm{d}$ & $\mathrm{s} / \mathrm{d}$ \\
\hline Lee \& Sinatra (2012) & Estudiantes universitarios & $\begin{array}{l}\text { Lectura de una historia } \\
\text { ficticia }\end{array}$ & $\begin{array}{l}\text { Al menos una } \\
\text { historia }\end{array}$ & $\begin{array}{l}\text { Al menos } \\
\text { una hora }\end{array}$ \\
\hline $\begin{array}{l}\text { Harackiewicz et al. } \\
\text { (2012); Hyde et al. } \\
(2016) ; \text { Rozek et al. } \\
(2015,2017)\end{array}$ & $\begin{array}{l}\text { Madres, padres y } \\
\text { estudiantes secundarios. }\end{array}$ & $\begin{array}{l}\text { Folleto en papel (1) y } \\
\text { electrónicos (1), sitios } \\
\text { web cuestionarios } \\
\text { electrónicos (1), } \\
\text { entrevistas telefónicas }\end{array}$ & s/d & $\mathrm{s} / \mathrm{d}$ \\
\hline Durik et al. (2014) & Estudiantes secundarios & Ensayos (1) & $\mathrm{s} / \mathrm{d}$ & 6 semanas \\
\hline Brown et al. (2015) & Estudiantes universitarios & $\begin{array}{l}\text { Artículos académicos, } \\
\text { encuestas }\end{array}$ & $\begin{array}{l}\text { Un párrafo (Estudio } 1 \\
\text { y 2), cuatro párrafos } \\
\text { (Estudio 3) }\end{array}$ & $\begin{array}{l}\text { Una clase al } \\
\text { menos }\end{array}$ \\
\hline $\begin{array}{l}\text { Harackiewicz et al. } \\
\text { (2016) }\end{array}$ & Estudiantes universitarios & Ensayos (1) & Al menos una página & 4 semestres \\
\hline
\end{tabular}

Nota. s/d: sin datos, el artículo no brindaba la información necesaria. 


\section{Intervenciones sobre utilidad percibida y sus efectos}

Si bien existen intervenciones tendientes a modificar la utilidad percibida por los estudiantes (e.g., Hulleman \& Harackiewicz, 2009; Lee Johnson \& Sinatra, 2012; Shechter et al., 2011) algunas de ellas no informan los efectos de las intervenciones sobre la percepción (Hyde et al., 2016; Lee Johnson \& Sinatra, 2012; Rozek, Svoboda, Harackiewicz, Hulleman, \& Hyde, 2017; Shechter et al., 2011), con lo cual no se puede discriminar los efectos de la intervención sobre la utilidad percibida. En contraste, existen otras intervenciones (Brown, Smith, Thoman, Allen, \& Muragishi, 2015; Durik, Schmidt, Shumow, \& Rodenbeck, 2014; Harackiewicz, Rozek, Hulleman, \& Hyde, 2012; Harackiewicz et al., 2016; Hulleman et al., 2010; Hulleman \& Harackiewicz, 2009; Hulleman, Kosovich, Barron, \& Daniel, 2017; Rozek, Hyde, Svoboda, Hulleman, \& Harackiewicz, 2015) que sí han informado de forma discriminada los efectos de sus intervenciones sobre la utilidad percibida y cómo, posteriormente, esta utilidad percibida ha generado modificaciones en otras variables.

En la tabla 2 se muestran las intervenciones sobre utilidad percibida y sus efectos sobre otras variables consecuentes. Como puede observarse en la tabla, las intervenciones han controlado el rol moderador de la motivación y el rendimiento previo de los estudiantes o nivel educativo de sus padres. En general, las intervenciones tuvieron efectos moderados y han mejorado la percepción de utilidad, el interés y el rendimiento referido a contenidos de materias y carreras de ciencias naturales, tecnología, ingeniería y matemáticas.

En muestras con estudiantes de nivel secundario, una investigación evaluó si las actividades áulicas que promovieran que los estudiantes de noveno y décimo grado conecten los materiales de aprendizaje con sus vidas podrían incrementar su motivación y aprendizaje. La intervención logró que aquellos que poseían bajas expectativas de éxito se interesaran más por las ciencias al final de semestre. Sin embargo, aquellos que ya tenían altas expectativas de éxito no revelaron modificaciones en cuanto a su interés en ciencias (Hulleman \& Harackiewicz, 2009).

En relación a estudios que han recurrido a muestras con estudiantes universitarios, se han encontrado efectos de la utilidad sobre variables como el interés, el rendimiento y la motivación. Concretamente, existe evidencia que indica que una intervención en la cual se manipula la relevancia de una actividad para la vida de los estudiantes incide sobre el interés, el rendimiento y la utilidad percibida, especialmente para aquellos estudiantes que poseen bajas expectativas de desempeño (Harackiewicz et al., 2016; Hulleman et al., 2010).

También, algunos estudios han encontrado que manipular la frecuencia con la cual los estudiantes realizan conexiones entre el material de estudio y sus vidas impacta sobre el interés de los mismos hacia el aprendizaje (Hulleman et al. 2017). Además, algunos autores han hallado que tras una intervención que enfatiza el valor de utilidad comunitario que posee un conocimiento particular, los estudiantes reportan mayores niveles de motivación hacia el aprendizaje de dicho conocimiento (Brown et al., 2015).

\section{Hacer conexiones: el mecanismo de las intervenciones en utilidad percibida}

En conjunto, los estudios han demostrado que las intervenciones diseñadas para aumentar la utilidad percibida posteriormente aumentaron el interés y el rendimiento, y que estos efectos son particularmente ciertos para aquellos estudiantes más vulnerables (para revisiones ver Durik, Hulleman, \& Harackiewicz, 2015; Harackiewicz, Tibbetts, Canning, \& Hyde, 2014; Hulleman et al., 2017). Un aspecto común de las intervenciones es la relación o conexión que los estudiantes hacen entre un tema y su propia vida; es importante tratar de entender cuál sería el motivo por el que, al efectuar tales conexiones, se produce una mejora en la utilidad percibida. Cabe aclarar que, aunque Hulleman et al. (2010), Hulleman y Harackiewicz (2009), y Harackiewicz et al. (2016) han usado los términos relevancia y valor de utilidad de manera intercambiable al describir sus intervenciones, existe una distinción importante que se debe hacer. Mientras que el valor de utilidad se refiere a la utilidad para una meta proximal o distal, la relevancia se refiere a la presencia de una relación entre un tema o idea y otro tema o idea, la cual podría incluir una meta, pero también incluye un conjunto más amplio de relaciones. Por ejemplo, las matemáticas podrían ser útiles porque me 
ayudarán en un trabajo futuro (valor de utilidad), o podrían relacionarse con mi vida porque los cajeros de la tienda lo necesitan incluso si no las utilizo (relevancia). Debido a que la intervención de valor de utilidad es un tipo de intervención de relevancia, un posible mecanismo para los efectos de intervención de valor de utilidad es que alentar a los estudiantes a encontrar una conexión les permite advertir relaciones que antes no tenían en mente. Hacer tales conexiones puede permitir a los individuos ver información nueva desde una perspectiva diferente y desarrollar una integra- ción más profunda de sus conocimientos (Bransford \& Schwartz, 1999). Por otra parte, hacer una conexión puede mejorar el aprendizaje al instigar un conjunto de procesos que generan un enfoque diferente capaz de aumentar el aprendizaje. Por ejemplo, si un estudiante encuentra una conexión personal durante una conferencia de psicología, el estudiante puede estar más interesado en la lectura asignada y discutir el material con amigos. En general, el estudiante puede estar más motivado para procesar activamente el material durante la conferencia y más tarde al leer el libro.

Talola 2

Intervenciones sobre utilidad percibida y efectos sobre otras variables

\begin{tabular}{|c|c|c|}
\hline Autores & Covariables & Variables dependientes \\
\hline Hulleman \& Harackiewicz (2009) & $\begin{array}{l}\text { Expectativas de éxito, interés en } \\
\text { ciencia }\end{array}$ & $\begin{array}{l}\text { Interés en ciencia }(\beta d=-.11) \text {, } \\
\text { rendimiento }(\beta d=-.18) \text {, Interés en } \\
\text { cursos de ciencia }\left(\beta d=.58 .{ }^{1}\right)\end{array}$ \\
\hline Hulleman et al. (2010) & Interés, expectativas de desempeño & $\begin{array}{l}\text { Interés situacional }(\beta \mathrm{d} 1=.66, \beta \mathrm{d} 2=.61) \text {, } \\
\text { Interés sostenido }(\beta \mathrm{d} 1=.60, \beta \mathrm{d} 2=.31) \text {, } \\
\text { Calificaciones }\left(\beta \mathrm{d} 2=.33 .{ }^{2}\right)\end{array}$ \\
\hline Harackiewicz et al. (2012) & Nivel educativo de los padres & Toma de cursos STEM $(\beta d=.18)$ \\
\hline Durik et al. (2014) & $\begin{array}{l}\text { Interés en ciencia inicial, } \\
\text { expectativas de éxito, desempeño } \\
\text { en ciencia, utilidad percibida inicial, } \\
\text { metas de logro }\end{array}$ & Interés en ciencia $(\beta d=.14)$ \\
\hline Brown et al. (2015) & & $\begin{array}{l}\text { Positividad hacia la investigación ( } \beta \mathrm{d} 1 \\
=.62, \beta \mathrm{i} 2=.64, \beta \mathrm{i} 3=.61) \text {, Motivación } \\
\text { hacia carreras futuras }(\beta \mathrm{d} 1=.33, \beta \mathrm{i} 2 \\
=.34, \beta \mathrm{i} 3=.44) \text {, Importancia de la } \\
\text { investigación }(\beta \mathrm{d} 2=.60, \beta \mathrm{d} 3=.26)\end{array}$ \\
\hline Rozek et al. (2015) & Nivel educativo de los padres & $\begin{array}{l}\text { Valoración futura de carreras STEM ( } \beta \mathrm{d} \\
=.29, \beta \mathrm{i}=.37 .{ }^{3} \text { ) }\end{array}$ \\
\hline Harackiewicz et al. (2016) & $\begin{array}{l}\text { Confianza en el rendimiento, } \\
\text { promedio de calificaciones }\end{array}$ & $\begin{array}{l}\text { Desempeño }(\beta d=.08) \text {, compromiso con } \\
\text { el material de estudio }(\beta d=.11)\end{array}$ \\
\hline Hulleman et al. (2017) & & Interés $\left(\beta \mathrm{d} 2=.37 .^{4}\right)$ \\
\hline
\end{tabular}

Nota. ${ }^{1}$. Covariación negativa entre los efectos de la intervención y las expectativas de éxitos reportadas inicialmente por los estudiantes; ${ }^{2}$. Las distinciones en los efectos sobre las variables $(1,2,3)$ refieren a cada estudio reportado en el artículo; ${ }^{3}$ Efecto indirecto mediado por la percepción que tenía el estudiante de la valoración que poseían sus padres hacía carreras STEM; ${ }^{4}$ La distinción en los efectos sobre la variable interés (1, 2) refieren a cada estudio reportado en el artículo 
El establecimiento de relaciones entre el conocimiento nuevo y las ideas antiguas puede crear una arquitectura cognitiva más rica que el estudiante pueda utilizar al estudiar. Como resultado, es más probable que los estudiantes que hacen más conexiones entre el material del curso y el conocimiento existente encuentren utilidad en el curso, lo que puede mejorar la motivación. En efecto, Hulleman et al. (2017) pusieron a prueba y demostraron positivamente la hipótesis de que la frecuencia de conexión es un mecanismo importante a través del cual la intervención de valor de utilidad tiene sus efectos. En sus estudios, los estudiantes que informaron haber generado conexiones con mayor frecuencia entre el material del curso y sus vidas informaron más interés en el material al final del semestre. Además, este vínculo entre la frecuencia de conexión y los resultados se explicó por un aumento concomitante en las percepciones del valor de utilidad. Así, la frecuencia de las conexiones es un aspecto importante para encontrar valor y desarrollar interés en el contenido académico. Específicamente, hacer conexiones frecuentes entre el material y la vida de los estudiantes aumenta el valor de utilidad y los resultados de aprendizaje.

\section{Conclusiones}

La actividad de los docentes $\mathrm{y}$, particularmente de los docentes universitarios, se encuentra con nuevos y persistentes desafíos, tales como la masificación de las aulas, las altas tasas de reprobación y deserción, la formación en competencias para el futuro, entre otros (Echeverría Samanes, \& Martínez Clares, 2018; González-Arias \& Martínez-Molina, 2016). Numerosos factores estratégicos, motivacionales y afectivos del estudiante han sido identificados como causas o condicionantes de los procesos de aprendizaje y sus resultados (Curione, Gründler, Píriz, \& Huertas, 2017; Dominguez-Lara, Calderón-De la Cruz, Alarcón-Parco, \& Navarro-Loli, 2017; Dominguez-Lara \& Merino-Soto, 2018; Sánchez-Rosas, Aguirre, Bovina-Martijena, \& Galarza, 2019). Aun cuando estos factores estén ligados a la experiencia estudiantil, los docentes se preocupan por determinar hasta qué punto pueden im- plementar prácticas que atenúen los efectos de los mismos en los resultados de aprendizaje.

Como se expuso en este artículo, distintas investigaciones han avanzado en la delimitación de las acciones que pueden aplicarse para mejorar la utilidad que los estudiantes perciben del aprendizaje (Hulleman \& Harackiewicz, 2009). La revisión de las actividades implementadas en cada intervención permite señalar que, dependiendo de la disponibilidad de tiempo y recursos técnicos, en general las mismas son simples y factibles. Las instrucciones que acompañan a las actividades son variadas, pero apuntan a un objetivo común: los estudiantes deben establecer relaciones significativas entre los temas de aprendizaje y su vida (Hulleman et al., 2017). Concretamente, las intervenciones consisten en pedirle a los estudiantes que relacionen los temas aprendidos en clase con su vida cotidiana, la búsqueda de información en sitios web sobre los temas tratados en clase, o el uso de ejemplos por parte del docente y padres que permita demostrar la utilidad de los contenidos para poder llevar a cabo una carrera universitaria a futuro. La realización de asociaciones entre el aprendizaje y la vida cotidiana y la frecuencia de dichas asociaciones podría ser el mecanismo por el cual las intervenciones impactan de forma positiva sobre la utilidad percibida. Teniendo en cuenta la factibilidad de manipular la utilidad percibida (Harackiewicz et al., 2016) y la simplicidad de los recursos necesarios (Durik et al., 2014; Harackiewicz et al., 2016; Hulleman et al., 2017; Hulleman \& Harackiewicz, 2009) para implementar las intervenciones, se considera que las mismas se deberían incorporar en la práctica docente.

Las intervenciones revisadas se caracterizan por dirigirse a estudiantes de nivel secundario y universitario, y los resultados de las mismas impactan de forma más positiva en aquellos estudiantes que poseen menores niveles de motivación y un menor desempeño. Asimismo, algunas intervenciones han mejorado la percepción de utilidad que poseen los estudiantes sobre contenidos de materias relacionadas a ciencias naturales, matemáticas e ingenierías (Brown et al., 2015; Durik et al., 2014; Harackiewicz et al., 2012; Hulleman \& Harackiewicz, 2009; Rozek et al., 2015). Teniendo en cuenta lo anterior, se considera que este conjunto de investigaciones aporta evidencia empíri- 
ca que podría ser de apoyo para la toma de decisiones de aquellas personas que generan políticas educativas. De esta manera, quienes gestionan las instituciones educativas alcanzarían un triple logro anhelado: innovar, motivar, y comprometer.

A pesar de las bondades que la evidencia revisada muestra, cabe señalar que las investigaciones analizadas presentan limitaciones. Existe consenso acerca de que los adolescentes presentan una disminución de su motivación en general (Rosenzweig \& Wigfield, 2016), por lo que se beneficiarían de las acciones tendientes a atenuar este problema. Si bien los estudios revisados indicarían que gracias a las intervenciones sería posible una transición motivada de los estudiantes de secundaria a carreras universitarias de ciencias naturales y tecnología, sería necesario evaluar si estas son igualmente efectivas para otras áreas del conocimiento. Otra limitación a considerar es que, si bien algunos procedimientos incluyeron la participación de las familias en la modificación de la utilidad percibida mediante los diálogos entre estudiantes de secundaria y sus padres, estas estrategias no fueron validadas para el nivel universitario, con lo cual sería relevante indagar el alcance de este tipo de intervenciones en poblaciones universitarias.

Por otra parte, aunque las investigaciones indagaron la problemática de la motivación en estudiantes de nivel universitario de grado, cabe mencionar que junto a la expansión de los posgrados en los últimos años también se extendieron tales problemas motivacionales a este nivel de formación (Bair \& Haworth, 2004). Por ello, los investigadores podrían poner a prueba la efectividad de sus intervenciones, analizando la capacidad de las mismas para incidir sobre la decisión de cursar, persistir o abandonar estudios de posgrado. Finalmente, es importante señalar que solo las variables interés, rendimiento y elección de carrera son analizadas de manera sistemática en los estudios. Distintos autores han identificado que variables tales como la atención o la participación en clase juegan un papel relevante en el proceso de aprendizaje (p.e., Molinari \& Sánchez-Rosas, 2018; Sánchez-Rosas, \& Esquivel, 2016), con lo cual se considera relevante incluir otras variables e indagar los efectos de las intervenciones en valor de utilidad sobre las mismas.
En síntesis, a pesar de que se requiere continuar indagando si estos resultados son generalizables a otros contextos educativos (Rosenzweig, Hulleman, Barron, Kosovich, Priniski, \& Wigfield, 2019), la implementación de intervenciones como las revisadas en este estudio podría mejorar la experiencia áulica retroalimentando positivamente las dinámicas de enseñanza-aprendizaje.

\section{Referencias}

Anderman, E. M., \& Maehr, M. L. (1994). Motivation and schooling in the middle grades. Review of Educational Research, 64(2), 287-309. https://doi. org/10.3102/00346543064002287

Bair, C. R., \& Haworth, J. G. (2004). Doctoral student attrition and persistence: a meta-synthesis of research. En J.C. Smart (Ed.), Higher education: Handbook of theory and research (pp. 481-534). The Netherlands: Kluwer Academic Publishers. https://doi. org/10.1007/1-4020-2456-8_11

Bransford, J. D., \& Schwartz, D. L. (1999). Rethinking transfer: A simple proposal with multiple implications. Review of Research in Education, 24, 61-100. https://doi.org/10.2307/1167267

Brown, E. R., Smith, J. L., Thoman, D. B., Allen, J. M., \& Muragishi, G. (2015). From bench to bedside: A communal utility value intervention to enhance students` biomedical science motivation. Journal of Educational Psychology, 107(4), 1116-1135. https:// doi.org/10.1037/edu0000033

Canning, E. A., Harackiewicz, J. M., Priniski, S. J., Hecht, C. A., Tibbetts, Y., \& Hyde, J. S. (2018). Improving performance and retention in introductory biology with a utility-value intervention. Journal of Educational Psychology, 110(6), 834-849. http://dx.doi. org/10.1037/edu0000244

Cook, J. E., Purdie-Vaughns, V., Garcia, J., \& Cohen, G. L. (2012). Chronic threat and contingent belonging: Protective benefits of values affirmation on identity development. Journal of Personality and Social Psychology, 102(3), 479-496. https://doi. org/10.1037/a0026312

Curione, K., Gründler, V., Píriz, L., \& Huertas, J. A. (2017). MSLQ-UY, validación con estudiantes universita- 
rios uruguayos. Revista Evaluar, 17(2), 1-17. https:// doi.org/10.35670/1667-4545.v17.n2.18716

Dominguez-Lara, S., Calderón-De la Cruz, G., Alarcón-Parco, D., \& Navarro-Loli, J. (2017). Relación entre Ansiedad ante exámenes y rendimiento en exámenes en universitarios: Análisis preliminar de la diferencia según asignatura. Revista Digital de Investigación en Docencia Universitaria, 11(1), 123 135. https://doi.org/10.19083/ridu.11.492

Dominguez-Lara, S., \& Merino-Soto, C. (2018). Cognitive Emotional Regulation Questionnaire-18 en Universitarios: Evidencias de Validez Convergente y Discriminante. Revista Iberoamericana de Diagnóstico y Evaluación Psicológica, 47(2). https://doi. org/10.21865/ridep47.2.12

Durik, A. M., \& Harackiewicz, J. M. (2007). Different strokes for different folks: How individual interest moderates the effects of situational factors on task interest. Journal of Educational Psychology, 99(3), 597-610. https://doi.org/10.1037/0022-0663.99.3.597

Durik, A. M., Hulleman, C. S., \& Harackiewicz, J. M. (2015). One Size Fits Some: Instructional enhancements to promote interest. En, K. A. Reninger, M. Niewswan$\mathrm{dt}$, \& S. Hidi (Eds.), Interest in Mathematics and Science Learning (pp. 49-62). Washington, DC: American Educational Research Association. https://doi.org/10.3102/978-0-935302-42-4_3

Durik, A. M., Schmidt, J. M., Shumow, L., \& Rodenbeck, B. (2014). Self-Generated utility among a diverse sample of adolescent students: An analysis of grade level and gender. Recuperado de: https://www. semanticscholar.org/paper/Self-Generated-Utility-Among-a-Diverse-Sample-of-\%3A-Durik-Schmi$\mathrm{dt} / \mathrm{f039ae32b3e5180fa3d69edeec9aa1c8dc7e541f}$

Durik, A. M., Vida, M., \& Eccles, J. S. (2006). Task values and ability beliefs as predictors of high school literacy choices: A developmental analysis. Journal of Educational Psychology, 98(2), 382-393. https://doi. org/10.1037/0022-0663.98.2.382

Eccles J. S., Adler, T. F., Futterman, R., Goff, S. B., Kaczala, C. M., Meece, J. L., \& Midgley, C. (1983). Expectancies, values, and academic behaviors. En, J. T. Spence (Ed.), Achievement and achievement motivation (pp. 75-146). San Francisco, CA: W. H. Freeman.

Echeverría Samanes, B., \& Martínez Clares, P. (2018). Revolución 4.0, competencias, educación y orientación. Revista Digital de Investigación en Docencia Universitaria, 12(2), 4-34. https://doi.org/10.19083/ ridu.2018.831
González-Arias, M. \& Martínez-Molina, A. (2016). Calidad docente, formación y evaluación de estudiantes universitarios: editorial. Revista de Psicología, 25(2), 1-3.

https://doi.org /10.5354/0719-0581.2017.44918

Harackiewicz, J. M., Canning, E. A., Tibbetts, Y., Priniski, S. J., \& Hyde, J. S. (2016). Closing achievement gaps with a utility-value intervention: Disentangling race and social class. Journal of Personality and Social Psychology, 111(5), 745-765. https://doi. org/10.1037/pspp0000075

Harackiewicz, J. M., Durik, A. M., Barron, K. E., Linnenbrink-Garcia, L., \& Tauer, J. M. (2008). The role of achievement goals in the development of interest: Reciprocal relations between achievement goals, interest, and performance. Journal of Educational Psychology, 100(1), 105-122. https://doi. org/10.1037/0022-0663.100.1.105

Harackiewicz, J. M., Rozek, C. S., Hulleman, C. S., \& Hyde, J. S. (2012). Helping parents to motivate adolescents in mathematics and science: and experimental test of a utility-value intervention. Psychological Science, 23(8), 899-906. https://doi. org/10.1177/0956797611435530

Harackiewicz, J. M., Tibbetts, Y., Canning, E., \& Hyde, J. S. (2014). Harnessing values to promote motivation in education. En, S. A. Karabenick, \& T. C. Urdan (Eds.), Advances in motivation and achievement (pp. 71-105). Bingley, UK: Emerald Group Publishing Limited. http://dx.doi.org/10.1108/S0749742320140000018002

Hattie, J. A.C. (2008). Visiblelearning: A synthesis of over 800 meta-analyses relating to achievement. London: Routledge. https://doi.org/10.4324/9780203887332

Hattie, J. A. C. (2012). Visible learning for teachers. London: Routledge. https://doi.org/10.4324/9780203181522

Hulleman, C. S., \& Harackiewicz, J. M. (2009). Promoting interest and performance in high school science Classes. Science, 329(5958), 1410-1412. https://doi. org/10.1126/science.1177067

Hulleman, C. S., Godes, O., Hendricks, B. L., \& Harackiewicz, J. M. (2010). Enhancing interest and performance with a utility value intervention. Journal of Educational Psychology, 102(4), 880-895. https://doi. org/10.1037/a0019506

Hulleman, C. S., Kosovich, J. J., Barron, K. E., \& Daniel, D. B. (2017). Making connections: replicating and extending the utility value intervention in the classroom. Journal of Educational Psychology, 109(3), 
387-404. https://doi.org/10.1037/edu0000146

Hyde, J. S., Canning, E. A., Rozek, C. S., Clarke, E., Hulleman, C. S., \& Harackiewicz, J. M. (2016). The role of mothers' communication in promoting motivation for math and science course-taking in high school. Journal of research on adolescence, 27(1), 49-64. https://doi.org/10.1111/jora.12253

Karadag, E. (2017). The factors effecting student achievement. Turquía: Springer Cham. https://doi. org/10.1007/978-3-319-56083-0

Lazowski R. A., \& Hulleman C. S. (2016). Motivation interventions in education. A meta-analytic review. Review of Educational Research, 86(2), 602-640. https://doi.org/10.3102/0034654315617832

Lee Johnson, M., \& Sinatra, G. M. (2012). Use of task-value instructional inductions for facilitating engagement and conceptual change. Contemporary Educational Psychology, 38(1), 51-63. https://doi. org/10.1016/j.cedpsych.2012.09.003

Lepper, M. R., Corpus, J. H., \& Iyengar, S. S. (2005). Intrinsic and extrinsic motivational orientations in the classroom: Age differences and academic correlates. Journal of Educational Psychology, 97(2), 184196. https://doi.org/10.1037/0022-0663.97.2.184https://doi.org/10.1037/0022-0663.97.2.184

Molinari, A., \& Sánchez Rosas, J. (2018). Compromiso comportamental: propuestas para la evaluación de participación social académica. Contextos de Educación, 20(25), 90-10.

Organización para la Cooperación y el Desarrollo Económicos. (2015). PISA 2015. Resultados clave. Recuperado de: https://www.oecd.org/pisa/pisa-2015-results-in-focus-ESP.pdfhttps://www.oecd.org/pisa/ pisa-2015-results-in-focus-ESP.pdf

Rosenzweig, E. Q., \& Wigfield, A. (2016). STEM Motivation Interventions for Adolescents: A Promising Start, but Further to Go. Educational Psychologist, 51(2), 146163. https://doi.org/10.1080/00461520.2016.1154792

Rosenzweig, E. Q., Harackiewicz, J. M., Priniski, S. J., Hecht, C. A., Canning, E. A., Tibbetts, Y., \& Hyde, J. S. (2018). Choose your own intervention: Using choice to enhance the effectiveness of a utility-value intervention. Motivation Science, publicación avanzada en línea. https://doi.org/10.1037/mot0000113

Rosenzweig, E. Q., Hulleman, C. S., Barron, K. E., Kosovich, J. J., Priniski, S. J., \& Wigfield, A. (2019). Promises and Pitfalls of Adapting Utility Value Interventions for Online Math Courses. The Journal of Experimental Education, 87(2), 332-352. https://doi.org/10.1080/

\subsection{8 .1496059}

Rozek, C. S., Hyde, J. S., Svoboda, R. C., Hulleman, C. S., \&, Harackiewicz, J. M. (2015). Gender differences in the effects of a utility-value intervention to help parents motivate adolescents in mathematics and science. Journal of Educational Psychology, 107(1), 195-206. https://doi.org/10.1037/a0036981

Rozek, C. S., Svoboda, R. C., Harackiewicz, J. M., Hulleman, C. S., \&, Hyde, J. S. (2017). Utility-value intervention with parents increases students' STEM preparation and career pursuit. Proceeding of the National Academy of Sciences, 114(5), 909-914. https://doi.org/10.1073/pnas.1607386114https://doi. org/10.1073/pnas.1607386114

Sánchez-Rosas, J., \& Esquivel, S. (2016). Instructional Teaching Quality, Task Value, SelfEfficacy, and Boredom: A Model of Attention in Class. Revista de Psicología, 25(2), 1-20. https://doi.org/10.5354/0719. 0581.2017 .44966

Sánchez-Rosas, J., Aguirre, R. S., Bovina-Martijena, N., \& Galarza, V. L. (2019). Motivational regulation strategies: A questionnaire for its measurement in Argentinian university students. Revista Evaluar, 19(1), 35-42. https://doi.org/10.35670/1667-4545.v19. $\mathrm{n} 1.23878$

Secretaria de Evaluación Educativa. Ministerio de Educación. (2017). APRENDER 2017 Informe de resultados secundaria. Recuperado de: https://www. argentina.gob.ar/sites/default/files/reporte_nacional_2017_secundaria_2.pdf

Shechter, O., Durik, A., Miyamoto, Y., \& Harackiewicz, J. (2011). The role of utility value in achievement behavior: The importance of culture. Personality and Social Psychology Bulletin, 37(3), 303-317. https:// doi.org/10.1177/0146167210396380

Shin, D.-J. D., Lee, M., Ha, J. E., Park, J. H., Ahn, H. S., Son, E., Chung Y., \& Bong, M. (2019). Science for all: Boosting the science motivation of elementary school students with utility value intervention. Learning and Instruction, 60, 104-116. https://doi. org/10.1016/j.learninstruc.2018.12.003

Simpkins, S. D., Davis-Kean, P. E., \& Eccles, J. S. (2006). Math and science motivation: A longitudinal examination of the links between choices and beliefs. Developmental Psychology, 42(1), 70-83. https://doi. org/10.1037/0012-1649.42.1.70

Walton, G. M. (2014). The new science of wise psychological interventions. Current Directions in Psychological Science, 23(1), 73- 82. https://doi. 
org/10.1177/0963721413512856

Wigfield, A., \& Cambria, J. (2010). Expectancy-value theory: Retrospective and prospective. En, T. C. Urdan, \& S.

A. Karabenick (Eds.), The decade ahead: Theoretical perspectives on motivation and achievement. Advances in motivation and achievement (pp. 35 70). Bingley, UK: Emerald Group Publishing Limited. https://doi.org/10.1108/S0749-7423(2010)000016A005

Wigfield, A., \& Eccles, J. S. (2000). Expectancy-Value theory of achievement motivation. Contemporary Educational Psychology, 25(1), 68-81. https://doi. org/10.1006/ceps.1999.1015

Xiang, P., Bruene, A., \& Chen, A. (2005). Interactive impact of intrinsic motivators and extrinsic rewards on behavior and motivation outcomes. Journal of Teaching in Physical Education, 24(2), 179-197. https:// doi.org/10.1123/jtpe.24.2.179

RIDU / Revista Digital de Investigación en Docencia Universitaria / e-ISSN: 2223-2516

(c) Los autores. Este artículo es publicado por la Revista Digital de Investigación en Docencia Universitaria del Área de Institutional Research and Effectiveness de la Dirección de Aseguramiento de la Calidad, Universidad Peruana de Ciencias Aplicadas. Este es un artículo de acceso abierto, distribuido bajo los términos de la LicenciaCreativeCommons Atribución-CompartirIgual 4.0 Internacional.(http://creativecommons.org/licenses/by-sa/4.0/), que permite el uso no comercial, distribución y reproducción en cualquier medio, siempre que la obra original sea debidamente citada. 